\title{
The Association between Habitual Sleep Duration and Sleep Quality with Glycemic Traits: Assessment by Cross-Sectional and Mendelian Randomization Analyses
}

\author{
Maxime M. Bos ${ }^{1, *} \mathbb{D}$, Diana van Heemst ${ }^{1}$ (D) Esther Donga $^{2}$, Renée de Mutsert ${ }^{3}$, \\ Frits R. Rosendaal ${ }^{3,4}$, Gerard Jan Blauw ${ }^{1}$, Patrick C. N. Rensen ${ }^{4,5}{ }^{\infty}$, Nienke R. Biermasz ${ }^{5}$ \\ and Raymond Noordam ${ }^{1, *}$ \\ 1 Department of Internal Medicine, section of Gerontology and Geriatrics, Leiden University Medical Center, \\ P.O. Box 9600, 2300 RC Leiden, The Netherlands; d.van_heemst@lumc.nl (D.v.H.); g.j.blauw@lumc.nl (G.J.B.) \\ 2 Departments of Radiology and Internal Medicine, Elisabeth TweeSteden Hospital, Hilvarenbeekseweg 60, \\ 5022 GC Tilburg, The Netherlands; e.donga@etz.nl \\ 3 Department of Clinical Epidemiology, Leiden University Medical Center, P.O. Box 9600, \\ 2300 RC Leiden, The Netherlands; r.de_mutsert@lumc.nl (R.d.M.); f.r.rosendaal@lumc.nl (F.R.R.) \\ 4 Einthoven Laboratory for Experimental Vascular Medicine, Leiden University Medical Center, P.O. Box 9600, \\ 2300 RC Leiden, The Netherlands; p.c.n.rensen@lumc.nl \\ 5 Department of Medicine, Division of Endocrinology, Leiden University Medical Center, P.O. Box 9600, \\ 2300 RC Leiden, The Netherlands; n.r.biermasz@lumc.nl \\ * Correspondence: m.m.bos@lumc.nl (M.M.B.); r.noordam@lumc.nl (R.N.)
}

Received: 28 March 2019; Accepted: 13 May 2019; Published: 15 May 2019

\begin{abstract}
Evidence on whether habitual sleep duration and sleep quality are associated with increased insulin resistance is inconsistent. Here, we investigated the associations between different measures of habitual sleep with glycemic traits through cross-sectional and Mendelian randomization (MR) analyses. We assessed the associations of sleep duration and sleep quality with glycemic traits using multivariable linear regression models adjusted for potential confounders in 4672 middle-aged (45-65 years; $48 \%$ men) nondiabetic participants of the Netherlands Epidemiology of Obesity (NEO) study. Genetic variants for total, short, and long sleep duration were used as instrumental variables in MR analyses using summary-level data of glycemic traits in nondiabetic individuals (MAGIC; $n=58,074$ ). In cross-sectional analyses, shortest sleepers (median $5.0 \mathrm{~h}$ of sleep per night) had 14.5\% (95\% confidence interval (CI): $2.0 ; 28.6 \%$ ) higher fasting insulin level and $16.3 \%$ ( $95 \%$ CI: $2.7 ; 31.7 \%$ ) higher HOMA- $\beta$. Bad sleep quality was associated with higher insulin resistance (e.g., 14.3\% (95\% CI: 4.7; 24.9\%) higher HOMA-IR). All these associations disappeared after adjustment for BMI and the risk of sleep apnea. MR analyses did not indicate a causal association between total, short or long sleep duration and glycemic traits. Therefore, our used measures of habitual sleep duration and sleep quality are unlikely to directly associate with insulin resistance.
\end{abstract}

Keywords: sleep; diabetes; epidemiology; insulin resistance; mendelian randomization

\section{Introduction}

During the past few decades, obesity and conditions that reflect disturbances in metabolism have increased [1]. Multiple studies have assessed the association between measures of habitual sleep and metabolic diseases. In several studies, it has been observed that both short and long total sleep duration were associated with a higher risk of obesity, insulin resistance, diabetes mellitus, and a higher body weight [2-9]. However, other studies reported only short sleep duration and not long sleep duration to 
be associated with a higher risk of obesity and metabolic syndrome $[6,10,11]$. As total sleep duration has decreased over the past decades [12], studies in this area are of increasing importance.

Several factors could contribute to the discrepancy in the observed associations between sleep duration and insulin resistance. For example, most studies investigated only short sleep duration [2], or short sleep duration under artificial circumstances [2,13], which might not resemble habitual short sleep duration. Moreover, cut-offs to define either short or long sleep duration are heterogeneous [14]. Another explanation might be adjustments for confounding factors. Body mass index (BMI) is known on one hand to be one of the largest risk factors for insulin resistance and diabetes, while on the other hand, it is associated with shorter sleep duration [15-17]. In a study in adolescents, an association between shorter sleep duration and insulin resistance was observed; however, this association disappeared after adjustment for BMI [18]. Although related to BMI, another potential confounding factor is the presence of obstructive sleep apnea (OSA). The presence of OSA was demonstrated to be associated with a higher risk of insulin resistance and diabetes [19]. However, most of the previous performed studies did not adjust for OSA [4,10,11,20-22].

The question remains as to what extent the previously described associations between sleep duration with insulin resistance were confounded by BMI and OSA. In addition to sleep duration, poor sleep quality is associated with a higher presence of obesity, metabolic syndrome, and diabetes [23,24]. However, studies examining poor sleep quality have mainly been performed in patients with diabetes $[5,24,25]$. If assessed in nondiabetic individuals, artificially altered sleep quality was studied by, e.g., suppressing slow-wave sleep [26], which does not resemble habitual poor sleep quality. Moreover, methods to assess sleep quality differed between studies. For example, some studies used only a single question about difficulty of initiating or maintaining sleep [5], while others used the Pittsburgh sleep quality index (PSQI) questionnaire [25] or other questionnaires [27,28]. These different methods to assess sleep quality complicate comparability among these studies.

Based on earlier studies, we hypothesized that both short sleep duration and bad sleep quality are associated with higher insulin resistance, but this association is likely dependent on anthropometric traits like BMI and the risk of sleep apnea. We aimed to examine this hypothesis in a middle-aged nondiabetic population embedded in the Netherlands Epidemiology of Obesity (NEO) study [29]. Furthermore, we extended this study with a two-sample Mendelian randomization (MR) analysis to provide evidence as to whether the association between sleep duration and insulin resistance is causal using data of the Meta-Analyses of Glucose and Insulin-Related Traits Consortium (MAGIC).

\section{Experimental Section}

\subsection{Study Design and Study Population}

The Netherlands Epidemiology of Obesity (NEO) study is a population-based cohort study. Participants were recruited from September 2008 until September 2012, resulting in a cohort of 6671 individuals, with an oversampling of individuals with overweight or obesity. Men and women aged between 45 and 65 years with a self-reported body mass index (BMI) of $27 \mathrm{~kg} / \mathrm{m}^{2}$ or higher living in the greater area of Leiden were eligible to participate in the NEO study. In addition, all inhabitants aged between 45 and 65 years from one municipality (Leiderdorp) were invited irrespective of their BMI, allowing for a reference distribution of BMI. Baseline data were collected at the NEO study center of the Leiden University Medical Center (LUMC). Prior to the NEO study visit, participants completed a questionnaire about demographic and clinical information and fasted for at least $10 \mathrm{~h}$. Participants came to the research site in the morning to undergo several baseline measurements, including anthropometric measurements and blood sampling. The participants drank a liquid mixed meal, after which postprandial blood sampling was performed. All medication used in the month preceding the visit to the study center was recorded by research nurses. More detailed information on the study design and data collection has been described elsewhere [29]. The NEO study was approved 
by the medical ethics committee of the LUMC (P08.109), and has been registered under NCT03410316 (https://clinicaltrials.gov). All participants provided written informed consent.

In the present study, we excluded participants with missing data on the PSQI questionnaire $(n=1402)$, which were collected in participants enrolled in NEO after July 2009. Moreover, we excluded participants that used glucose lowering medication $(n=263)$, had a medical history of diabetes mellitus ( $n=77)$, had missing baseline characteristics $(n=132)$, had missing glucose or insulin concentrations $(n=48)$, had missing data on the Berlin questionnaire $(n=57)$ or did not fast $(n=20)$. In the analyses with the postprandial measures, we additionally excluded participants with incomplete or no liquid meal intake $(n=8)$ and participants with either missing postprandial glucose $(n=153)$ or postprandial insulin concentrations $(n=78)$.

\subsection{Sleep Characteristics}

To assess habitual sleep duration and sleep quality, we used data collected with the PSQI [30], which is a self-rated questionnaire, to retrospectively measure sleep parameters over a one-month time period. Total sleep duration was derived from the question: "On an average night, how much sleep do you get?" To obtain a classification of (extreme) short and long total sleep duration independent of age and sex, we calculated the age- and sex-adjusted residuals with linear regression analysis for total sleep duration and determined subgroups on the basis of these residuals, as previously described [17,31]. We used the 5th lowest percentile to define shortest sleep, the 5th to the 20th percentile to define short sleep, the 20th to the 80th to define medium sleep, the 80th to the 95th to define long sleep, and the 95th to the 100th percentile to define longest sleep of the adjusted residuals. Sleep quality was assessed using the total score of the PSQI questionnaire. The questionnaire consists of seven components, based on which an overall score can be calculated ranging from 0 to 21 , in which a higher score indicates poorer sleep quality [30]. In the sleep quality analyses, the good sleep quality group (PSQI total score $\leq 5$ ) was used as a reference group in linear regression analyses with two groups with either a PSQI total score of $>5$ or $\geq 10$, the latter in order to investigate a more extreme disturbed habitual sleep.

\subsection{Glycemic Traits}

After an overnight fast of at least $10 \mathrm{~h}$, fasting blood samples were taken at the study center. Within $5 \mathrm{~m}$ after the first blood sample was taken, participants drank a liquid mixed meal. This meal $(400 \mathrm{~mL})$ contained $600 \mathrm{kcal}$, with $16 \%$ of energy derived (En\%) from protein, $50 \%$ En $\%$ from carbohydrates, and $34 \mathrm{En} \%$ from fat. Two postprandial blood samples were taken at 30 and $150 \mathrm{~m}$ after ingestion of the mixed meal. Serum and plasma were collected during each of the three blood draws, and concentrations of glucose and insulin were determined. Fasting plasma glucose concentrations were determined by enzymatic and colorimetric methods (Roche Modular Analytics P800, Roche Diagnostics, Mannheim, Germany; $\mathrm{CV}<5 \%$ ), and serum insulin concentrations were determined by an immunometric method (Siemens Immulite 2500, Siemens Healthcare Diagnostics, Breda, The Netherlands; CV $<5 \%$ ). All analyses were performed in the central clinical chemistry laboratory of the Leiden University Medical Center. Fasting glucose and insulin levels were used to calculate the homeostatic model assessment of insulin resistance (HOMA-IR) index as a marker for hepatic insulin resistance. The HOMA-IR was calculated using (fasting insulin $\times$ fasting glucose)/22.5. HOMA of $\beta$-cell function (HOMA- $\beta$ ) was used as a marker that indicates basal insulin release and calculated as $20 \times$ (fasting insulin/fasting glucose) $-3.5[32,33]$. The area under the curve (AUC) for postprandial overall glucose and insulin levels was calculated using the trapezoid rule as $\left(15 \times\right.$ fasting concentration $+75 \times$ concentration $_{30 \mathrm{~min}}$ $+60 \times$ concentration $\left._{150 \mathrm{~min}}\right) / 150$ [34].

\subsection{Covariates}

Level of education was reported in 10 categories according to the Dutch education system and grouped into high versus low education [29]. A semiquantitative food frequency questionnaire 
(FFQ) [35] questionnaire was used to assess daily total energy intake. Energy intake was estimated from the FFQ with the 2011 version of the Dutch food composition table (NEVO-2011). Participants reported the frequency and duration of their physical activity in leisure time using the short questionnaire to assess health-enhancing physical activity (SQUASH) [36], which was expressed in hours per week of metabolic equivalents (MET-h/week). Body weight was measured without shoes, and one kilogram $(\mathrm{kg})$ was subtracted to correct for the weight of clothing. BMI was calculated by dividing the weight in kilograms by the height in meters squared. The Berlin questionnaire was used to assess the risk for the presence of obstructive sleep apnea syndrome [37]. This questionnaire consists of 10 questions that form three categories (snoring (category 1), daytime somnolence (category 2), and hypertension and BMI (category 3)) related to the likelihood of the presence of sleep apnea. Individuals can be classified as either having a high ( 2 or more categories with a positive score) or low likelihood of the presence of sleep apnea (only 1 or no categories with a positive score).

\subsection{Statistical Analysis}

Because individuals with a BMI of $27 \mathrm{~kg} / \mathrm{m}^{2}$ or higher were oversampled in the NEO study, adjustments were made to correctly represent associations in the general population [38]. This was done by weighting individuals towards the BMI distribution of participants from the Leiderdorp municipality, whose BMI distribution was similar to the BMI distribution of the general Dutch population. All presented results are based on weighted analyses. Consequently, the results apply to a population-based study without oversampling of participants with a BMI of $27 \mathrm{~kg} / \mathrm{m}^{2}$ or higher. We performed all statistical analyses of the NEO cohort using Stata version 12.1 (Stata, College Station, TX, USA).

Baseline characteristics were expressed as means (with standard deviation, SD) for normally distributed measures, median with interquartile ranges for non-normally distributed measures, and proportions for categorical variables.

Not normally distributed outcomes were log-transformed to approximate a normal distribution (notably fasting insulin, HOMA-IR, HOMA- $\beta$, AUC insulin). In order to present the results with a similar interpretation, normally distributed outcomes (notably fasting glucose and AUC glucose) were log-transformed as well. All outcome variables were approximately normally distributed after log-transformation, including those that were already normally distributed prior to the transformation. Linear regression analyses using the medium sleep category (characterized by the 20th to the 80th percentile of sleep duration residuals) as a reference group were performed. The subsequent beta regression coefficients are expressed as percentages with accompanying 95\% confidence interval ( $95 \% \mathrm{CI})$, which can be interpreted as the percentage change in outcome with respect to the reference group. The initial model in linear regression analyses was adjusted for age and sex (Model 1). In addition to age and sex, we adjusted Model 2 for ethnicity (white/other), education level (high/other), smoking (never/former/current), alcohol consumption (g/day), energy intake (kJ/day), physical activity (MET/h/week), and sleep medication (yes/no). In Model 3, we additionally adjusted for sleep apnea (high risk/low risk) and BMI $\left(\mathrm{kg} / \mathrm{m}^{2}\right)$. All selected covariables were only low to moderately correlated with each other (Supplementary Figure S1). In the analyses for sleep quality, we did not adjust for sleep medication in Models 2 and 3, as this is a component of the PSQI total score.

\subsection{Mendelian Randomization Analysis}

For the MR analysis, we selected 78 single nucleotide polymorphisms (SNPs) that have been shown to associate with self-reported total sleep duration $\left(p\right.$-value $\left.<5 \mathrm{e}^{-8}\right)$ as genetic instruments from the largest genome-wide association study (GWAS; source: UK Biobank, 446,118 unrelated European-ancestry individuals) [39] (Supplementary Table S4). Moreover, we selected 27 SNPs associated with short sleep duration ( $<7 \mathrm{~h} ; n=106,192$ cases) and 8 SNPs associated with long sleep duration ( $\geq 9 \mathrm{~h} ; n=34,184$ cases) relative to $7-8 \mathrm{~h}$ of sleep duration $(n=305,742)$ (Supplementary Tables S5 and S6). 
Summary statistics data of GWAS on glycemic traits were used as outcomes in a two-sample MR approach. We used data from MAGIC, which comprised a meta-analysis of European ancestry studies that investigated genetic variants associated with glycemic traits. For fasting glucose and fasting insulin, we used the GWAS of Manning et al. (2012) [40], which included a total of 58,074 and 51,570 individuals without diabetes mellitus, respectively. In addition, we used HOMA-IR and HOMA-B as measures of insulin resistance $(n=46,186)$ [41].

For the two-sample MR analyses, we used similar methodology as described previously [42]. In short, we combined effects of the individual genetic instruments using the inverse variance weighted (IVW) approach as our main analysis method with MRCIEU/TwoSampleMR package in $\mathrm{R}$ [43]. The resulting estimate (presented with accompanying 95\% CI) can be interpreted as a weighted mean effect of a genetically determined increase in total sleep duration (per hour) and a higher risk for short or long sleep duration on our study outcomes. In order to formally test for potential pleiotropic effects of the genetic variants, in which the genetic variants have pleiotropic effects that influence glycemic traits via alternative pathways, we conducted MR-Egger regression as sensitivity analysis [44]. We furthermore conducted weighted median estimator (WME) analyses; similarity between the IVW and WME effect estimates is indicative of robustness of the results [45].

\section{Results}

\subsection{Baseline Characteristics}

In the present study, we included a total of 4672 participants with a mean age of 56 (SD 6.0) years, of whom $48 \%$ were men. In Table 1, the population characteristics are presented for the study population, stratified by the sleep duration groups. As compared to the medium sleep group (sleep duration $=7 \mathrm{~h} /$ day), participants in the shortest sleep group (sleep duration $=5 \mathrm{~h}$ /day) were more of nonwhite ancestry ( $96 \%$ vs. $90 \%$ ), had a lower education ( $50 \%$ high vs. $39 \%$ high), higher BMI ( 26 vs. $\left.27 \mathrm{~kg} / \mathrm{m}^{2}\right)$, smoked more (16\% vs. 18\%), were less physically active (31 vs. $25 \mathrm{MET} / \mathrm{h} /$ week), used more sleep medication ( $4 \%$ vs. $14 \%$ ), had a higher PSQI total score (4 vs. 11$)$, and had a higher risk of having sleep apnea (17\% vs. $35 \%$ ). In the shortest sleep group, fasting insulin, HOMA- $\beta$, and AUC of insulin were higher than in the medium sleep group. As compared to the medium group, the longest sleep duration group consisted of more men ( $42 \%$ vs. $53 \%)$, more current smokers ( $16 \%$ vs. $20 \%$ ), fewer high-educated participants ( $50 \%$ vs. $36 \%$ ), more frequent use of sleep medication ( $4 \%$ vs. $7 \%$ ), and more participants with a higher risk of having sleep apnea ( $17 \%$ vs. $24 \%)$ as compared to the medium sleep group. All other characteristics were similar between the groups.

\subsection{Sleep Duration and Glycemic Traits}

In the analyses adjusted for age and sex (Model 1; explained variance up to 7.4), shortest sleep was associated with a $17.9 \%(95 \%$ CI: $4.8 ; 32.5 \%)$ higher fasting insulin, an $18.4 \%$ (95\% CI: $3.9 ; 34.9 \%)$ higher HOMA-IR, an $18.8 \%$ (95\% CI: 4.9; 34.7\%) higher HOMA- $\beta$, and a $17.5 \%$ (95\% CI: 7.9; $28.0 \%$ ) higher AUC for insulin as compared to the medium sleep group (Figure 1 and Supplementary Table S1). When we adjusted for potential confounding factors (Model 2; explained variance up to $8.4 \%$ ), shortest sleep duration was associated with a $14.5 \%(95 \%$ CI: $2.0 ; 28.6 \%)$ higher fasting insulin, a $14.9 \%(95 \%$ CI: 1.0 ; $30.7 \%$ ) higher HOMA-IR, a $16.3 \%$ (95\% CI: $2.7 ; 31.7 \%$ ) higher HOMA- $\beta$, and a $14.5 \%$ (95\% CI: $4.9 ; 24.9 \%)$ higher AUC of insulin. However, the associations between shortest sleep duration and HOMA-IR attenuated when analyses were adjusted for considered potential confounding factors (Model 2). When we additionally adjusted for BMI and risk of sleep apnea (Model 3; explained variance up to $29.5 \%$ ) the other associations observed for shortest sleep duration disappeared. However, in Model 3, longest sleep was associated with a $-16.3 \%$ (95\% CI: $-29.6 ;-0.4 \%)$ lower HOMA-IR as compared to the medium sleep duration group. The same attenuation of associations was observed when we adjusted separately for either BMI or the risk of sleep apnea (Supplementary Table S1: Models 3 and 4). 
Table 1. Characteristics of participants in the Netherlands Epidemiology of Obesity study, stratified by sleep duration $(n=4672)$.

\begin{tabular}{cccccc}
\hline Sleep Duration & Shortest & Short & Medium & Long & Longest \\
\hline & $\mathbf{0 - 5 \%}$ & $\mathbf{5 - 2 0 \%}$ & $\mathbf{2 0 - 8 0 \%}$ & $\mathbf{8 0 - 9 5 \%}$ & $\mathbf{9 5 - 1 0 0 \%}$ \\
\hline Age (years) & $57(5)$ & $58(5)$ & $55(6)$ & $54(6)$ & $58(6)$ \\
Sex (\% men) & 45 & 49 & 42 & 43 & 53 \\
BMI (kg/m $\mathbf{m}^{2}$ & $27(5)$ & $26(4)$ & $26(4)$ & $26(4)$ & $26(5)$ \\
Ethnicity (\% white) & 90 & 94 & 96 & 96 & 94 \\
Education (\% high) & 39 & 41 & 50 & 48 & 36 \\
Smoking (\%current) & 18 & 14 & 16 & 16 & 20 \\
Sleep medication (\%) & 14 & 10 & 4 & 5 & 7 \\
Alcohol consumption (g/day) & $12(3 ; 22)$ & $11(3 ; 22)$ & $10(3 ; 21)$ & $9(2 ; 21)$ & $11(1 ; 23)$ \\
Physical activity (MET/h/week) & $25(12 ; 44)$ & $30(16 ; 50)$ & $31(17 ; 51)$ & $32(15 ; 52)$ & $30(16 ; 49)$ \\
\hline Sleep duration (h/day) & $5(4 ; 5)$ & $6(6 ; 6)$ & $7(7 ; 8)$ & $8(8 ; 8)$ & $9(9 ; 9)$ \\
PSQI (total score) & $11(9 ; 13)$ & $7(5 ; 9)$ & $4(3 ; 6)$ & $3(1 ; 4)$ & $3(1 ; 5)$ \\
Sleep apnea (\%) & 35 & 25 & 17 & 17 & 24 \\
\hline Fasting glucose (mmol/L) & $6(1)$ & $6(2)$ & $6(1)$ & $6(1)$ & $6(2)$ \\
Fasting insulin (mmol/L) & $9(6 ; 14)$ & $8(6 ; 12)$ & $7(5 ; 11)$ & $7(5 ; 11)$ & $7(4 ; 12)$ \\
HOMA-IR & $2(1 ; 4)$ & $2(1 ; 3)$ & $2(1 ; 3)$ & $2(1 ; 3)$ & $2(1 ; 3)$ \\
HOMA- $\boldsymbol{\beta}$ & $28(18 ; 42)$ & $26(18 ; 40)$ & $24(16 ; 37)$ & $25(15 ; 36)$ & $25(13 ; 37)$ \\
AUC Glucose * & $6(1)$ & $6(1)$ & $6(1)$ & $6(1)$ & $6(1)$ \\
AUC Insulin ${ }^{*}$ & $47(34 ; 62)$ & $41(30 ; 57)$ & $38(29 ; 53)$ & $38(30 ; 54)$ & $41(26 ; 61)$ \\
\hline
\end{tabular}

Abbreviations: AUC, area under the curve; BMI, body mass index; HOMA- $\beta$, homeostatic model of assessment of $\beta$-cell function; HOMA-IR, homeostatic model of assessment insulin resistance; kJ, kilojoule; MET, metabolic equivalents of task; NEO, Netherlands Epidemiology of Obesity; PSQI, Pittsburgh sleep questionnaire index. Results were based on analyses weighted towards the BMI distribution of the general Dutch population. Data presented as mean \pm standard deviation (SD); proportion (\%); median (25th-75th percentile). ${ }^{*}, n=4511$; ${ }^{*} n=4586$.

A)

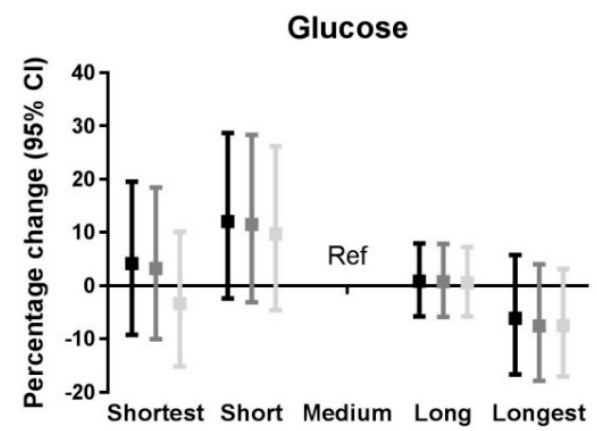

C)

HOMA-IR

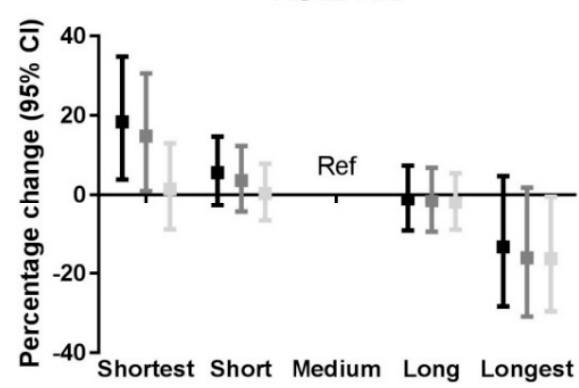

B)

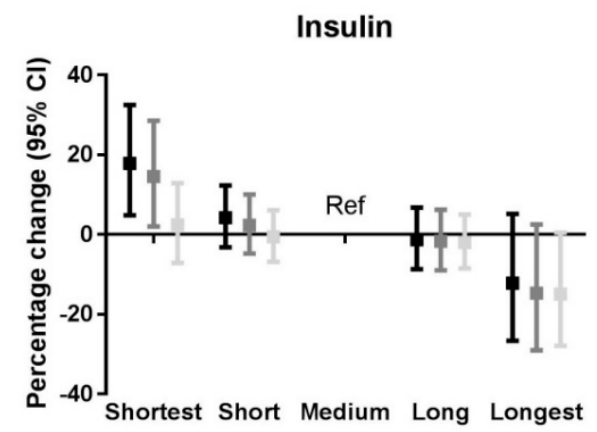

D)

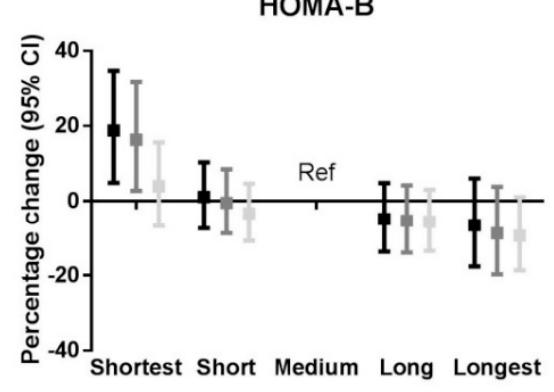

Figure 1. Cont. 
E)

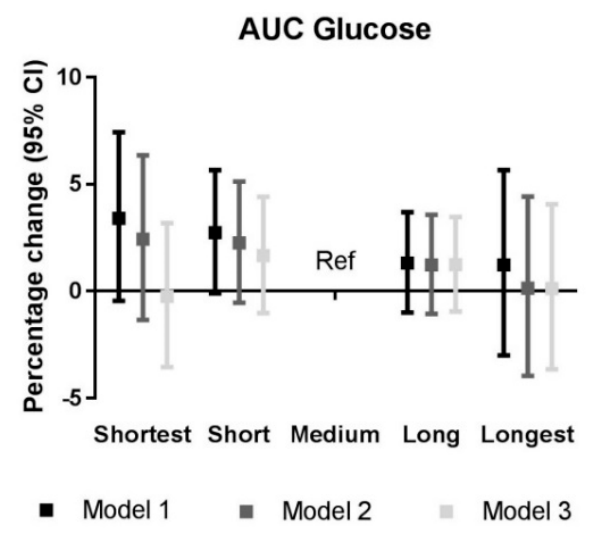

F)

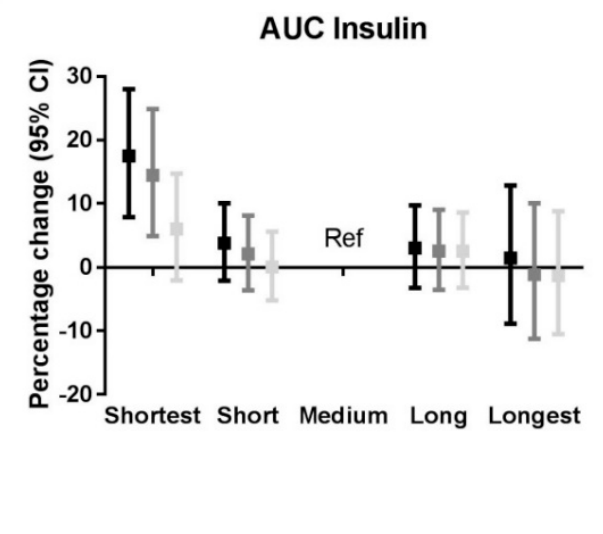

Figure 1. Associations between sleep duration and (A) fasting glucose, (B) fasting insulin, (C) HOMA-IR, (D) HOMA- $\beta$, (E) AUC of glucose, and (F) AUC of insulin. Results were based on analyses weighted towards the BMI distribution of the general Dutch population $(n=4672)$ and were derived from regression coefficients with $95 \%$ confidence intervals from linear regression analyses and expressed as percentage change in outcome measure, as compared to the medium sleep duration group as reference category. Model 1: Adjusted for age and sex; Model 2: Adjusted for age, sex, ethnicity, education level, smoking, alcohol intake, caloric intake, and physical activity; Model 3: Adjusted for Model $2+$ risk of sleep apnea and BMI. Abbreviations: AUC, area under the curve; CI, confidence interval; HOMA-IR, homeostatic model of insulin resistance; $\operatorname{HOMA}-\beta$, homeostatic model of $\beta$ cell function; Ref, reference category. $n=4511$ for the analyses with AUC glucose and $n=4586$ for the analyses with AUC insulin.

\subsection{Sleep Quality and Glycemic Traits}

The associations between sleep quality and glycemic traits are visualized in Figure 2 and Supplementary Table S2. We did find evidence for an association between poor sleep quality, as defined by a PSQI score of $>5$ and a 6.5\% (95\% CI: $0.0 ; 13.5 \%$ ) higher HOMA- $\beta$ in Model 1 (explained variance up to $7.1 \%$ ) as compared to the good sleep quality group (PSQI score $\leq 5$ ). However, when we adjusted for potential confounding factors in Model 2 (explained variance up to 8.0\%), this association disappeared (percentage change $5.1 \%(95 \% \mathrm{CI}$ : $-1.1 ;-11.8 \%)$. In the age- and sex-adjusted analyses (Model 1), a PSQI score $\geq 10$ was associated with a $16.9 \%$ (95\% CI: 7.6; $27.1 \%$ ) higher fasting insulin, a $17.3 \%$ (95\% CI: 7.0; $28.5 \%$ ) higher HOMA-IR, an $18.6 \%$ (95\% CI: 8.4; $29.7 \%)$ higher HOMA- $\beta$, and a $7.7 \%$ (95\% CI: $0.7 ; 15.2 \%$ ) higher AUC of insulin as compared to the good sleep quality group. When we additionally adjusted for potential confounding factors in Model 2, we observed a $14.1 \%$ (95\% CI: 5.3; $23.7 \%$ ) higher fasting insulin, a $14.3 \%$ (95\% CI: 4.7; 24.9\%) higher HOMA-IR, and a $15.7 \%(95 \%$ CI: $6.0 ; 26.4 \%$ ) higher HOMA- $\beta$ in the group with a PSQI total score of $\geq 10$ as compared to the good sleep quality group. However, all associations attenuated when we additionally adjusted for BMI and the risk of sleep apnea (Model 3; explained variance up to 29.2\%). These associations attenuated in a similar manner when we adjusted the model for only BMI or the risk of sleep apnea (Supplementary Table S2: Models 3 and 4). 
A)

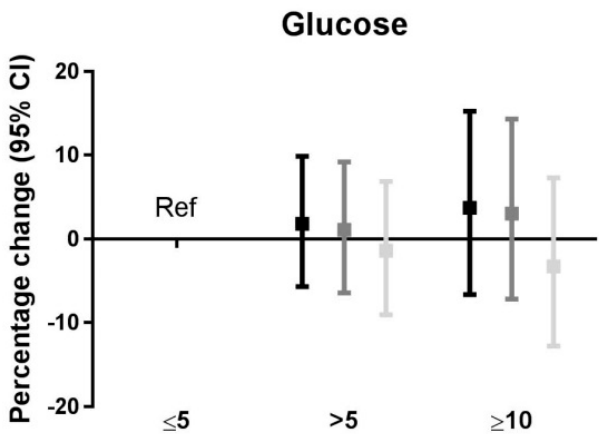

C)

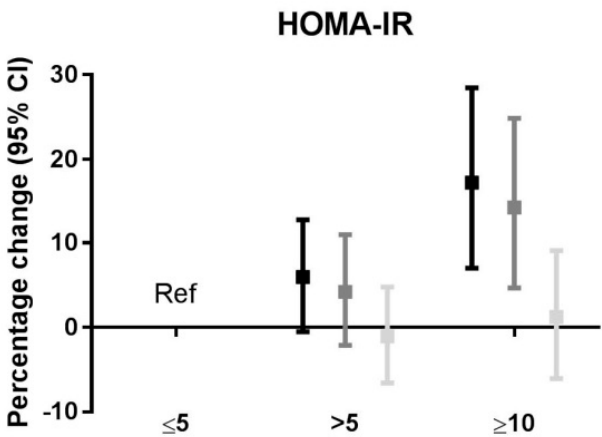

E)

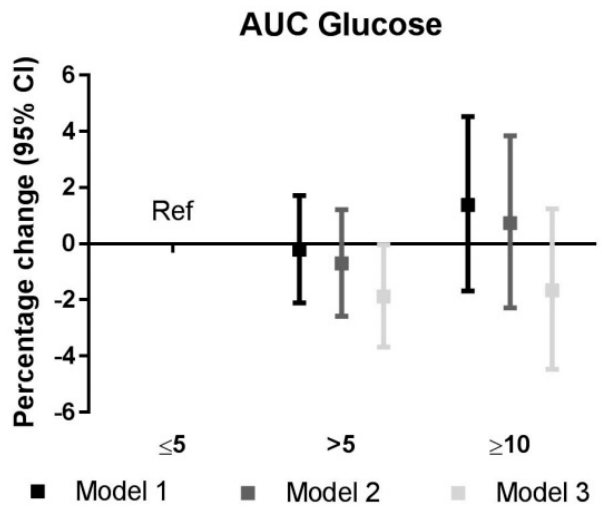

B)

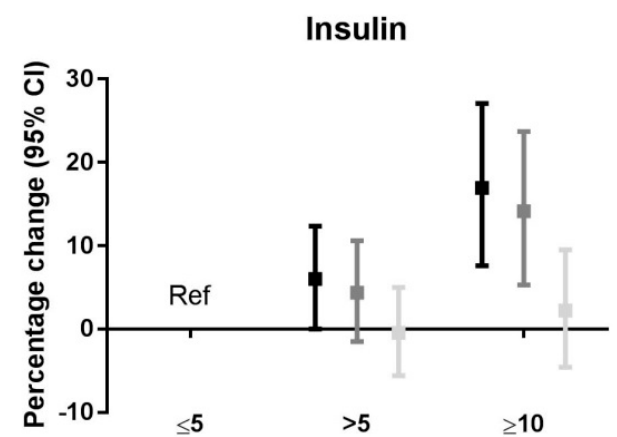

D)

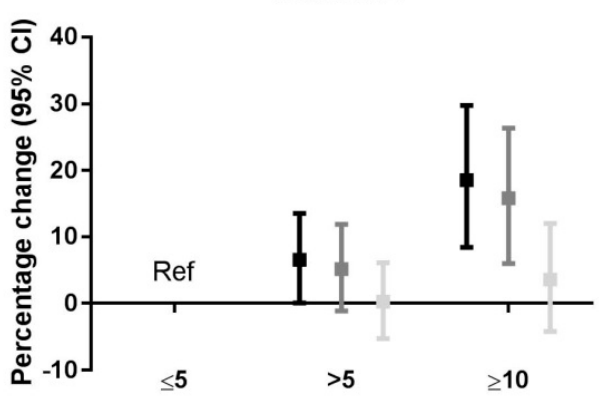

F)

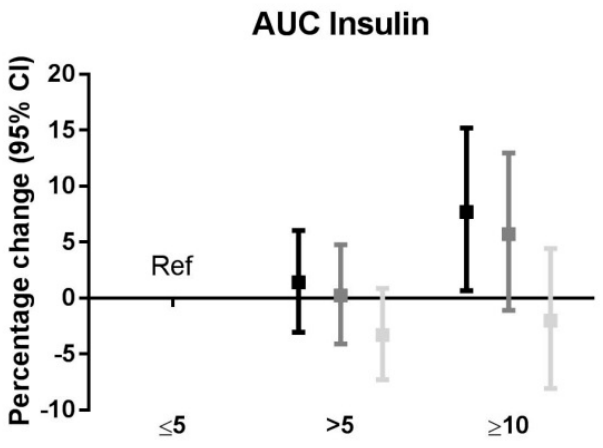

Figure 2. Associations between sleep quality and (A) fasting glucose, (B) fasting insulin, (C) HOMA-IR, (D) HOMA- $\beta$, (E) AUC of glucose, and (F) AUC of insulin. Results were based on analyses weighted towards the BMI distribution of the general Dutch population $(n=4672)$ and were derived from regression coefficients with $95 \%$ confidence intervals from linear regression analyses, using either a group with a PSQI total score of $>5$ or $\geq 10$, and expressed as percentage change in outcome measure, as compared to the good sleep group as reference category. Model 1: Adjusted for age and sex; Model 2: Adjusted for age, sex, ethnicity, education level, smoking, alcohol intake, caloric intake, and physical activity; Model 3: Adjusted for Model 2 + risk of sleep apnea and BMI. Abbreviations: AUC, area under the curve; CI, confidence interval; HOMA-IR, homeostatic model of insulin resistance; HOMA- $\beta$, homeostatic model of $\beta$ cell function; Ref, reference category. $n=4511$ for the analyses with AUC glucose and $n=4586$ for the analyses with AUC insulin.

\subsection{Genetically-Determined Habitual Sleep Duration and Glycemic Traits}

In order to assess whether the association observed between sleep duration and glycemic traits (Model 1 and 2) is causal, we performed an MR study. We found no evidence for an association 
between genetically determined total sleep duration, short sleep duration, and long sleep duration with glycemic traits using IVW-analyses (Table 2, Supplementary Table S3). The WME analyses were consistent with these results (Supplementary Table S3). However, we did find evidence for an association between total sleep duration and a higher HOMA- $\beta$ (IVW-estimate: 0.08 (95\% CI: 0.01; 0.014)). The estimate from WME analysis was consistent, and MR-Egger did not indicate the presence of directional pleiotropy in this analysis (Supplementary Table S3).

Table 2. Inverse-variance weighted estimates for sleep duration on glycemic traits.

\begin{tabular}{ccccccc}
\hline & & $\begin{array}{c}\text { Total Sleep } \\
\text { Duration }\end{array}$ & & $\begin{array}{c}\text { Short Sleep } \\
\text { Duration }\end{array}$ & & $\begin{array}{c}\text { Long Sleep } \\
\text { Duration }\end{array}$ \\
\hline & SNPs & Estimate (95\% CI) & SNPs & Estimate (95\% CI) & SNPs & Estimate (95\% CI) \\
\hline Fasting glucose & 54 & $-0.03(-0.11 ; 0.06)$ & 20 & $-0.09(-0.19 ; 0.01)$ & 5 & $-0.05(-0.24 ; 0.13)$ \\
Fasting insulin & 54 & $0.01(-0.04 ; 0.07)$ & 20 & $-0.01(-0.11 ; 0.08)$ & 5 & $-0.06(-0.36 ; 0.23)$ \\
HOMA-IR & 53 & $0.07(-0.01 ; 0.15)$ & 20 & $0.04(-0.08 ; 0.15)$ & 5 & $-0.01(-0.47 ; 0.45)$ \\
HOMA- $\beta$ & 53 & $0.08(0.01 ; 0.14)$ & 20 & $0.09(-0.01 ; 0.19)$ & 5 & $0.09(-0.26 ; 0.45)$ \\
\hline
\end{tabular}

Data presented as beta coefficients with $95 \%$ confidence interval per standard deviation increase in exposure.

\section{Discussion}

In the present study, we performed a cross-sectional analysis in a middle-aged population of 4519 nondiabetic participants to determine the associations between habitual sleep duration and sleep quality with glycemic traits. When analyses were adjusted for age and sex, shortest sleep duration was associated with higher fasting insulin, higher HOMA-IR, higher HOMA- $\beta$, and higher AUC of insulin. Poorer sleep quality was associated with higher fasting insulin, higher HOMA-IR, higher HOMA- $\beta$, and higher AUC of insulin. However, all these associations disappeared after additional adjustment for BMI and the risk of sleep apnea. Furthermore, in the MR analyses, we, overall, did not observe associations between genetically-determined total, short and long sleep duration, and glycemic traits. Of interest, we found some evidence that longer genetically-determined sleep duration was associated with a higher HOMA- $\beta$.

Previous studies showed that both short and long sleep duration, or only short sleep duration were associated with a higher risk of obesity, body weight, insulin resistance, and diabetes mellitus $[2-5,10,11]$. We hypothesized that these differences in findings may be explained by confounding factors such as BMI and sleep apnea. In agreement, in the age- and sex-adjusted analyses, we observed associations between shorter sleep duration and higher insulin resistance, which disappeared when we additionally adjusted for BMI and the presence of sleep apnea. Our findings are in agreement with a study of Javaheri et al., who also observed an association between short sleep duration and insulin resistance, which disappeared after adjustment for obesity [18]. In line, in our MR study, genetically-determined sleep duration was, in general, not associated with glycemic traits, indicating that there is no direct effect of sleep on glycemic traits. However, it has been observed that there is a shared genetic component between sleep and anthropometrics [46]. Moreover, adiposity has been shown to be causally associated with an increased risk of diabetes and altered glycemic traits [16]. Therefore, we hypothesize that excess adiposity is a common cause for alterations in habitual sleep and insulin resistance, instead of being in the causal path of this association.

An alternative explanation for these observations is that BMI/risk of sleep apnea is in the causal pathway between shorter sleep duration and increased insulin resistance. It was shown that sleep restriction reduces leptin levels and enhances ghrelin levels and thereby increases cravings for carbohydrate-rich foods, which may result in weight gain [47]. Another mechanism that links short sleep duration with insulin resistance might be via obstructive sleep apnea (OSA). It was demonstrated that patients with OSA showed increased insulin resistance compared to controls without OSA, independent of obesity [48]. These might indicate that, although obesity is known to effect insulin resistance [16], OSA may have an independent additional effect on insulin resistance, possibly via 
nocturnal hypoxemia [49]. A GWAS of Lane et al. on total sleep duration suggested that there is a shared genetic component between sleep duration and energy metabolism [46], which demonstrates that the genetic background of an individual may also interfere with altered sleep duration and energy metabolism.

One of the strengths of this study is the use of residuals to determine sleep duration. As no clear cut-off for short and long sleep exists in literature, using residuals is a good way to harmonize grouping of sleep duration among studies. Moreover, the heterogeneity of future meta-analyses and systematic reviews might benefit from using this methodology. Another strength of this study is the extensive phenotyping of the NEO study. This enables us to correct for a broad range of possible confounding factors, such as ethnicity, physical activity, BMI, and risk of sleep apnea. In addition, we used the PSQI questionnaire, which is a widely used validated tool to assess sleep disturbances. A limitation of the present study is the cross-sectional design; therefore, we are not able to exclude reverse causation or residual confounding in this study. Another limitation of this study is the use of subjective sleep questionnaires to assess sleep duration, sleep quality, and the risk of sleep apnea. One of the drawbacks of self-reported data is the fact that these data are subject to recall bias. Moreover, there may be a measurement error resulting in nondifferential misclassification for the exposure. Therefore, the use of a sleep diary may improve reliability of the measures of habitual sleep. However, the PSQI questionnaire has been shown to be a reliable and validated tool to assess sleep dysfunction, and the Berlin questionnaire, used to assess the risk of sleep apnea, has been shown to be validated to detect mild and severe OSA in several populations [50,51]. The MR study has the advantage of being a highly efficient method that allows for usage of large sample sizes; however, although we used the largest GWAS to date, we cannot rule out the use of weak instruments. The 78 loci used in the current study only explained $0.69 \%$ of the variance in sleep duration. Moreover, the number of genetic instruments that were present in this current study, especially for long sleep duration, was minimal. Finally, the identification of the genetic variants as well as the MR study were both performed in cohorts of European ancestry, which hampers the generalizability to non-European populations.

\section{Conclusions}

In summary, shorter sleep duration and poorer sleep quality were associated with higher insulin resistance, but these associations were dependent on BMI and the risk of sleep apnea. Both BMI and the risk of sleep apnea, as being potential confounding factors and/or factors in the causal pathway, thereby likely explain previous observed associations between adverse habitual sleep and an increased risk of insulin resistance, suggesting that these factors should therefore be considered in future studies.

Supplementary Materials: The following are available online at http://www.mdpi.com/2077-0383/8/5/682/s1, Table S1: Associations between sleep duration and glycemic traits, Table S2: Association between sleep quality and glycemic traits, Table S3: Mendelian Randomization estimates for sleep duration on glycemic traits, Table S4: Genetic variants for sleep duration, Table S5: Genetic variants for short sleep duration, Table S6: Genetic variants for long sleep duration, Figure S1: Correlation plot of studied variables.

Author Contributions: Conceptualization, M.M.B., R.N., E.D., N.R.B. and D.v.H.; methodology, M.M.B. and R.N.; software, M.M.B.; validation, M.M.B., R.d.M., P.C.N.R. and R.N.; formal analysis, M.M.B.; investigation, M.M.B., R.N., R.d.M. and F.R.R.; resources, R.d.M. and F.R.R.; data curation, M.M.B., G.J.B., D.v.H. and R.N.; writing—original draft preparation, M.M.B.; writing—review and editing, M.M.B., R.N., E.D., R.d.M., F.R.R., G.J.B., P.C.N.R., N.R.B. and D.v.H.; visualization, M.M.B.; supervision, R.N., D.v.H. and G.J.B.; project administration, M.M.B., R.N., D.v.H. and G.J.B.; funding acquisition, D.v.H.

Funding: This research was funded by the Netherlands Cardiovascular Research Initiative: An initiative with the support of the Dutch Heart Foundation (CVON2014-02 ENERGISE). D.v.H. was supported by the European Commission funded project HUMAN (Health-2013-INNOVATION-1-602757). N.R.B. was supported by the Netherlands Organization for Scientific Research (NWO-VENI 016.136.125). The NEO study is supported by the participating Departments, the Division, and the Board of Directors of the Leiden University Medical Centre, and by the Leiden University, Research Profile Area 'Vascular and Regenerative Medicine'. Nutricia Research, Utrecht, The Netherlands, provided the mixed meal.

Acknowledgments: We express our gratitude to all individuals who participated in the Netherlands Epidemiology of Obesity study. We are grateful to all participating general practitioners for inviting eligible participants. 
We furthermore thank P.R. van Beelen and all research nurses for collecting the data, P.J. Noordijk and her team for sample handling and storage, and I. de Jonge for all data management of the NEO study.

Conflicts of Interest: The authors declare no conflict of interest.

\section{References}

1. Gregg, E.W.; Shaw, J.E. Global Health Effects of Overweight and Obesity. N. Engl. J. Med. 2017, 377, 80-81. [CrossRef]

2. Wong, P.M.; Manuck, S.B.; Dinardo, M.M.; Korytkowski, M.; Muldoon, M.F. Shorter Sleep Duration is Associated with Decreased Insulin Sensitivity in Healthy White Men. Sleep 2015, 38, 223-231. [CrossRef] [PubMed]

3. Reutrakul, S.; Van Cauter, E. Interactions between sleep, circadian function, and glucose metabolism: Implications for risk and severity of diabetes. Ann. N. Y. Acad. Sci. 2014, 1311, 151-173. [CrossRef]

4. Cappuccio, F.P.; Taggart, F.M.; Kandala, N.-B.; Currie, A.; Peile, E.; Stranges, S.; Miller, M.A. Meta-Analysis of Short Sleep Duration and Obesity in Children and Adults. Sleep 2008, 31, 619-626. [CrossRef]

5. Cappuccio, F.P.; D’Elia, L.; Strazzullo, P.; Miller, M.A. Quantity and quality of sleep and incidence of type 2 diabetes: A systematic review and meta-analysis. Diabetes Care 2010, 33, 414-420. [CrossRef]

6. Liu, A.; Kushida, C.A.; Reaven, G.M. Habitual Shortened Sleep and Insulin Resistance: An Independent Relationship in Obese Individuals. Metab. Clin. Exp. 2013, 62, 1553-1556. [CrossRef]

7. Rutters, F.; Besson, H.; Walker, M.; Mari, A.; Konrad, T.; Nilsson, P.M.; Balkau, B.; Dekker, J.M. The Association Between Sleep Duration, Insulin Sensitivity, and $\beta$-Cell Function: The EGIR-RISC Study. J. Clin. Endocrinol. Metab. 2016, 101, 3272-3280. [CrossRef] [PubMed]

8. Van Dijk, D.; Balkau, B.; Segrestin, B.; Gottsäter, M.; Gabriel, R.; Hatunic, M.; Mari, A.; Dekker, J.M.; Rutters, F.; on behalf of the EGIR-RISC Study Group. Associations between sleep duration and sleep debt with insulin sensitivity and insulin secretion in the EGIR-RISC Study. Diabetes Metab. 2018. [CrossRef] [PubMed]

9. Brady, E.; Bodicoat, D.; Hall, A.; Khunti, K.; Yates, T.; Edwardson, C.; Davies, M. Sleep duration, obesity and insulin resistance in a multi-ethnic UK population at high risk of diabetes. Diabetes Res. Clin. Pract. 2018, 139, 195-202. [CrossRef] [PubMed]

10. Wu, Y.; Zhai, L.; Zhang, D. Sleep duration and obesity among adults: A meta-analysis of prospective studies. Sleep Med. 2014, 15, 1456-1462. [CrossRef] [PubMed]

11. Xi, B.; He, D.; Zhang, M.; Xue, J.; Zhou, D. Short sleep duration predicts risk of metabolic syndrome: A systematic review and meta-analysis. Sleep Med. Rev. 2014, 18, 293-297. [CrossRef] [PubMed]

12. A Schoenborn, C.; Adams, P.F.; A Peregoy, J. Health behaviors of adults: United States, 2008-2010. Health Stat. Ser. 10 2013, 10, 1-184.

13. Donga, E.; Van Dijk, M.; Van Dijk, J.G.; Biermasz, N.R.; Lammers, G.-J.; Van Kralingen, K.W.; Corssmit, E.P.M.; Romijn, J.A. A Single Night of Partial Sleep Deprivation Induces Insulin Resistance in Multiple Metabolic Pathways in Healthy Subjects. J. Clin. Endocrinol. Metab. 2010, 95, 2963-2968. [CrossRef]

14. Upala, S.; Sanguankeo, A.; Congrete, S.; Romphothong, K. Sleep duration and insulin resistance in individuals without diabetes mellitus: A systematic review and meta-analysis. Diabetes Res. Clin. Pract. 2015, 109, e11-e12. [CrossRef]

15. Kahn, S.E.; Hull, R.L.; Utzschneider, K.M. Mechanisms linking obesity to insulin resistance and type 2 diabetes. Nat. Cell Boil. 2006, 444, 840-846. [CrossRef] [PubMed]

16. Dale, C.E.; Fatemifar, G.; Palmer, T.M.; White, J.; Prieto-Merino, D.; Zabaneh, D.; Engmann, J.E.L.; Shah, T.; Wong, A.; Warren, H.R.; et al. Causal Associations of Adiposity and Body Fat Distribution with Coronary Heart Disease, Stroke Subtypes and Type 2 Diabetes: A Mendelian Randomization Analysis. Circulation 2017, 135, 2373-2388. [CrossRef]

17. Dekker, S.A.; Noordam, R.; Biermasz, N.R.; Roos, A.; Lamb, H.J.; Rosendaal, F.R.; Rensen, P.C.; Heemst, D.; Mutsert, R.; De Roos, A.; et al. Habitual Sleep Measures are Associated with Overall Body Fat, and not Specifically with Visceral Fat, in Men and Women. Obesity 2018, 26, 1651-1658. [CrossRef]

18. Javaheri, S.; Storfer-Isser, A.; Rosen, C.L.; Redline, S. Association of short and long sleep durations with insulin sensitivity in adolescents. J. Pediatr. 2011, 158, 617-623. [CrossRef] [PubMed] 
19. Morgenstern, M.; Wang, J.; Beatty, N.; Batemarco, T.; Sica, A.L.; Greenberg, H. Obstructive sleep apnea: An unexpected cause of insulin resistance and diabetes. Endocrinol. Metab. Clin. N. Am. 2014, 43, 187-204. [CrossRef]

20. Cappuccio, F.P.; Cooper, D.; D’Elia, L.; Strazzullo, P.; Miller, M.A. Sleep duration predicts cardiovascular outcomes: a systematic review and meta-analysis of prospective studies. Eur. Heart J. 2011, 32, 1484-1492. [CrossRef]

21. Berg, J.F.V.D.; Miedema, H.M.E.; Tulen, J.H.M.; Neven, A.K.; Hofman, A.; Witteman, J.C.M.; Tiemeier, H. Long Sleep Duration is Associated With Serum Cholesterol in the Elderly: The Rotterdam Study. Psychosom. Med. 2008, 70, 1005-1011. [CrossRef]

22. Shin, H.-Y.; Kang, G.; Kim, S.-W.; Kim, J.-M.; Yoon, J.-S.; Shin, I.-S. Associations between sleep duration and abnormal serum lipid levels: data from the Korean National Health and Nutrition Examination Survey (KNHANES). Sleep Med. 2016, 24, 119-123. [CrossRef]

23. Muldoon, M.F.; Hall, M.; Jennings, J.R.; Buysse, D.J.; Manuck, S.B. Self-reported Sleep Quality is Associated With the Metabolic Syndrome. Sleep 2007, 30, 219-223.

24. Koren, D.; Dumin, M.; Gozal, D. Role of sleep quality in the metabolic syndrome. Diabetes Metab. Syndr. Obes. Targets Ther. 2016, 9, 281-310. [CrossRef]

25. Tang, Y.; Meng, L.; Li, D.; Yang, M.; Zhu, Y.; Li, C.; Jiang, Z.; Yu, P.; Li, Z.; Song, H.; et al. Interaction of sleep quality and sleep duration on glycemic control in patients with type 2 diabetes mellitus. Chin. Med. J. 2014, 127, 3543-3547. [PubMed]

26. Tasali, E.; Leproult, R.; Ehrmann, D.A.; Van Cauter, E. Slow-wave sleep and the risk of type 2 diabetes in humans. Proc. Natl. Acad. Sci. USA 2008, 105, 1044-1049. [CrossRef]

27. Byberg, S.; Hansen, A.-L.S.; Christensen, D.L.; Vistisen, D.; Aadahl, M.; Linneberg, A.; Witte, D. Sleep duration and sleep quality are associated differently with alterations of glucose homeostasis. Diabet. Med. 2012, 29, e354-e360. [CrossRef]

28. Engeda, J.; Mezuk, B.; Ratliff, S.; Ning, Y. Association between duration and quality of sleep and the risk of pre-diabetes: Evidence from NHANES. Diabet. Med. 2013, 30, 676-680. [CrossRef] [PubMed]

29. De Mutsert, R.; Den heijer, M.; Rabelink, T.J.; Smit, J.W.; Romijn, J.A.; Jukema, J.W.; de Roos, A.; Cobbaert, C.M.; Kloppenburg, M.; le Cessie, S.; et al. The Netherlands Epidemiology of Obesity (NEO) study: Study design and data collection. Eur. J. Epidemiol. 2013, 28, 513-523. [CrossRef] [PubMed]

30. Buysse, D.J.; Reynolds, C.F., 3rd; Monk, T.H.; Berman, S.R.; Kupfer, D.J. The Pittsburgh Sleep Quality Index: A new instrument for psychiatric practice and research. Psychiatry Res. 1989, 28, 193-213. [CrossRef]

31. Noordam, R.; Bos, M.M.; Wang, H.; Winkler, T.W.; Bentley, A.R.; Kilpeläinen, T.; De Vries, P.S.; Sung, Y.J.; Schwander, K.; Cabe, B.E.; et al. Multi-ancestry analysis of gene-sleep interactions in 126,926 individuals identifies multiple novel blood lipid loci that contribute to our understanding of sleep-associated adverse blood lipid profile. bioRxiv 2019. [CrossRef]

32. Matthews, D.R.; Hosker, J.P.; Rudenski, A.S.; Naylor, B.A.; Treacher, D.F.; Turner, R.C. Homeostasis model assessment: insulin resistance and beta-cell function from fasting plasma glucose and insulin concentrations in man. Diabetologia 1985, 28, 412-419. [CrossRef] [PubMed]

33. Wallace, T.M.; Levy, J.C.; Matthews, D.R. Use and abuse of HOMA modeling. Diabetes Care 2004, 27, 1487-1495. [CrossRef]

34. Retnakaran, R.; Shen, S.; Hanley, A.J.; Vuksan, V.; Hamilton, J.K.; Zinman, B. Hyperbolic Relationship Between Insulin Secretion and Sensitivity on Oral Glucose Tolerance Test. Obesity 2008, 16, 1901-1907. [CrossRef] [PubMed]

35. Feunekes, G.I.; Van Staveren, W.A.; De Vries, J.H.; Burema, J.; Hautvast, J.G. Relative and biomarker-based validity of a food-frequency questionnaire estimating intake of fats and cholesterol. Am. J. Clin. Nutr. 1993, 58, 489-496. [CrossRef] [PubMed]

36. Wendel-Vos, G. Reproducibility and relative validity of the short questionnaire to assess health-enhancing physical activity. J. Clin. Epidemiol. 2003, 56, 1163-1169. [CrossRef]

37. Netzer, N.C.; Stoohs, R.A.; Netzer, C.M.; Clark, K.; Strohl, K.P. Using the berlin questionnaire to identify patients at risk for the sleep apnea syndrome. Ann. Intern. Med. 1999, 131, 485. [CrossRef] [PubMed]

38. Korn, E.L.; Graubard, I.B. Epidemiologic studies utilizing surveys: accounting for the sampling design. Am. J. Public Health 1991, 81, 1166-1173. [CrossRef] [PubMed] 
39. Dashti, H.S.; Redline, S.; Saxena, R. Polygenic risk score identifies associations between sleep duration and diseases determined from an electronic medical record biobank. Sleep 2018, 42. [CrossRef]

40. Manning, A.K.; DIAbetes Genetics Replication and Meta-analysis (DIAGRAM) Consortium; Hivert, M.-F.; Scott, R.A.; Grimsby, J.L.; Bouatia-Naji, N.; Chen, H.; Rybin, D.; Liu, C.-T.; Bielak, L.F.; et al. A genome-wide approach accounting for body mass index identifies genetic variants influencing fasting glycemic traits and insulin resistance. Nat. Genet. 2012, 44, 659-669. [CrossRef]

41. Upuis, J.; DIAGRAM Consortium; Langenberg, C.; Prokopenko, I.; Saxena, R.; Soranzo, N.; Jackson, A.U.; Wheeler, E.; Glazer, N.L.; Bouatia-Naji, N.; et al. New genetic loci implicated in fasting glucose homeostasis and their impact on type 2 diabetes risk. Nat. Genet. 2010, 42, 105-116. [CrossRef]

42. Bos, M.M.; Smit, R.A.J.; Trompet, S.; Van Heemst, D.; Noordam, R. Thyroid Signaling, Insulin Resistance, and 2 Diabetes Mellitus: A Mendelian Randomization Study. J. Clin. Endocrinol. Metab. 2017, 102, 1960-1970. [CrossRef] [PubMed]

43. Hemani, G.; Zheng, J.; Elsworth, B.; Wade, K.H.; Haberland, V.; Baird, D.; Laurin, C.; Burgess, S.; Bowden, J.; Langdon, R.; et al. The MR-Base platform supports systematic causal inference across the human phenome. eLife 2018, 7, e34408. [CrossRef] [PubMed]

44. Bowden, J.; Smith, G.D.; Burgess, S. Mendelian randomization with invalid instruments: effect estimation and bias detection through Egger regression. Int. J. Epidemiol. 2015, 44, 512-525. [CrossRef] [PubMed]

45. Bowden, J.; Smith, G.D.; Haycock, P.C.; Burgess, S. Consistent estimation in mendelian randomization with some invalid instruments using a weighted median estimator. Genet. Epidemiol. 2016, 40, 304-314. [CrossRef]

46. Lane, J.M.; Liang, J.; Vlasac, I.; Anderson, S.G.; Bechtold, D.A.; Bowden, J.; Emsley, R.; Gill, S.; Little, M.A.; Luik, A.I.; et al. Genome-wide association analyses of sleep disturbance traits identify new loci and highlight shared genetics with neuropsychiatric and metabolic traits. Nat. Genet. 2017, 49, 274-281. [CrossRef]

47. Spiegel, K.; Tasali, E.; Penev, P.; Van Cauter, E. Brief communication: Sleep curtailment in healthy young men is associated with decreased leptin levels, elevated ghrelin levels, and increased hunger and appetite. Ann. Intern. Med. 2004, 141, 846. [CrossRef] [PubMed]

48. Tassone, F.; Lanfranco, F.; Gianotti, L.; Pivetti, S.; Navone, F.; Rossetto, R.; Grottoli, S.; Gai, V.; Ghigo, E.; Maccario, M. Obstructive sleep apnoea syndrome impairs insulin sensitivity independently of anthropometric variables. Clin. Endocrinol. 2003, 59, 374-379. [CrossRef]

49. Dewan, N.A.; Nieto, F.J.; Somers, V.K. Intermittent hypoxemia and OSA: Implications for comorbidities. Chest 2015, 147, 266-274. [CrossRef]

50. Mollayeva, T.; Thurairajah, P.; Burton, K.; Mollayeva, S.; Shapiro, C.M.; Colantonio, A.; Information, P.E.K.F.C. The Pittsburgh sleep quality index as a screening tool for sleep dysfunction in clinical and non-clinical samples: A systematic review and meta-analysis. Sleep Med. Rev. 2016, 25, 52-73. [CrossRef] [PubMed]

51. Amra, B.; Rahmati, B.; Soltaninejad, F.; Feizi, A. Screening Questionnaires for Obstructive Sleep Apnea: An Updated Systematic Review. Oman Med. J. 2018, 33, 184-192. [CrossRef] [PubMed] 